\title{
Development and validation of a cancer stem cell-related signature for prognostic prediction in pancreatic ductal adenocarcinoma
}

\author{
Zengyu Feng ${ }^{1,2+}$, Minmin Shi ${ }^{1,2 \dagger}$, Kexian $\mathrm{Li}^{1,2}$, Yang Ma ${ }^{1,2}$, Lingxi Jiang ${ }^{1,2^{*}}$, Hao Chen ${ }^{1,2^{*}}$ \\ and Chenghong Peng ${ }^{1,2^{*}}$ (D)
}

\begin{abstract}
Background: Cancer stem cells (CSCs) are crucial to the malignant behaviour and poor prognosis of pancreatic ductal adenocarcinoma (PDAC). In recent years, CSC biology has been widely studied, but practical prognostic signatures based on CSC-related genes have not been established or reported in PDAC.

Methods: A signature was developed and validated in seven independent PDAC datasets. The MTAB-6134 cohort was used as the training set, while one local Chinese cohort and five other public cohorts were used for external validation. CSC-related genes with credible prognostic roles were selected to form the signature, and their predictive performance was evaluated by Kaplan-Meier survival, receiver operating characteristic (ROC), and calibration curves. Correlation analysis was employed to clarify the potential biological characteristics of the gene signature.

Results: A robust signature comprising DCBLD2, GSDMD, PMAIP1, and PLOD2 was developed. It classified patients into high-risk and low-risk groups. High-risk patients had significantly shorter overall survival (OS) and disease-free survival (DFS) than low-risk patients. Calibration curves and Cox regression analysis demonstrated powerful predictive performance. ROC curves showed the better survival prediction by this model than other models. Functional analysis revealed a positive association between risk score and CSC markers. These results had cross-dataset compatibility. Impact

This signature could help further improve the current TNM staging system and provide data for the development of novel personalized therapeutic strategies in the future.
\end{abstract}

Keywords: Pancreatic ductal adenocarcinoma, Cancer stem cell, Prognosis, Signature, Risk score

\section{Background}

Pancreatic ductal adenocarcinoma (PDAC) is an aggressive and lethal malignancy with a 5-year survival rate less than $9 \%[1,2]$. Surgical resection is the only curative

\footnotetext{
*Correspondence: j|x12120@rih.com.cn; Haochendr@126.com; chhpeng@yeah.net

†Zengyu Feng and Minmin Shi contributed equally to this work 1 Department of General Surgery, Pancreatic Disease Center, Research Institute of Pancreatic Diseases, Ruijin Hospital, Shanghai Jiaotong University School of Medicine, Shanghai, China

Full list of author information is available at the end of the article
}

treatment; unfortunately, a small minority of patients diagnosed with localized tumours are deemed eligible for curative surgery [3]. Patients without surgical indication can only receive adjuvant chemotherapy and radiotherapy [4]. These conventional treatments have improved the prognosis of advanced patients. However, in a given patient, their effects are questionable, and overtreatment can lead to adverse effects [5]. Currently, prognostic assessment and treatment decisions are mainly determined by the TNM staging system, which is not adequate for individual survival prediction, especially in

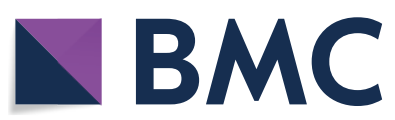

(c) The Author(s) 2020. This article is licensed under a Creative Commons Attribution 4.0 International License, which permits use, sharing, adaptation, distribution and reproduction in any medium or format, as long as you give appropriate credit to the original author(s) and the source, provide a link to the Creative Commons licence, and indicate if changes were made. The images or other third party material in this article are included in the article's Creative Commons licence, unless indicated otherwise in a credit line to the material. If material is not included in the article's Creative Commons licence and your intended use is not permitted by statutory regulation or exceeds the permitted use, you will need to obtain permission directly from the copyright holder. To view a copy of this licence, visit http://creativeco mmons.org/licenses/by/4.0/. The Creative Commons Public Domain Dedication waiver (http://creativecommons.org/publicdomain/ zero/1.0/) applies to the data made available in this article, unless otherwise stated in a credit line to the data. 
patients with the same tumour stage [6,7]. These problems highlight the need for a prognostic model to accurately predict patient survival and to guide the selection of reasonable treatment options.

Several previous studies have attempted to build risk prediction models by assessing critical biological processes associated with cancer, such as autophagy, epithelial-mesenchymal transition (EMT), and the DNA damage-repair pathway [8-10]. Similarly, cancer stem cells (CSCs), a small population of cancer cells (accounting for only $0.2-0.8 \%$ of PDAC cells) with self-renewal and multilineage differentiation capacities, have become promising therapeutic targets in PDAC treatment. They are responsible for tumour growth, invasion, metastasis, recurrence and therapeutic resistance $[11,12]$. Moreover, several CSC-specific markers, including CD9, CD24, CD44, and CD133, are highly expressed in PDAC tissues and significantly associated with poor patient prognosis [13-16]. Thus, CSC-related gene expression profiles can be used as a practical tool to predict prognosis.

In recent years, progress in next-generation sequencing technologies has allowed researchers to establish several prognostic signatures for PDAC [17-22]. All of these models have robust predictive ability, but they are not accurate enough, as most of them are derived from the single TCGA-PAAD dataset. A study showed that the failure to exclude non-PDAC samples from the TCGAPAAD cohort might lead to false conclusions regarding the prognostic value of biomarkers [23]. Furthermore, the inclusion a large number of genes (up to 36) hinders the translation of predictive models into clinical application. Therefore, a more concise and precise prognostic signature is urgently needed.

The aim of this study was to identify key CSC-related genes that are involved in PDAC development. By taking with survival information into account, we further investigated the prognostic role of these genes in multiple cohorts. Finally, we proposed a reliable four-gene signature for predicting both overall survival (OS) and disease-free survival (DFS), which can help prevent low-risk patients from experiencing the side effects of overtreatment.

\section{Materials and methods}

\section{Data source and processing}

$\mathrm{T}$ The gene expression profiles and related clinical data of patients were retrieved and downloaded from the Gene Expression Omnibus (GEO), ArrayExpress, International Cancer Genome Consortium (ICGC), and The Cancer Genome atlas (TCGA) databases. For the TCGA data, gene expression data were obtained from the TCGA hub at UCSC Xena (https://tcga.xenahubs.net). Normalized RNA-sequencing data for all available PACA-AU and
PACA-CA samples were downloaded from the ICGC data portal release 28. Microarray data were normalized using a robust multi-array averaging (RMA) method [24]. In each dataset, ineligible samples were excluded using the following criteria: (a) patients without complete clinical information; (b) non-PDAC samples such as pancreatic neuroendocrine neoplasms; and (c) PDAC cell lines, xenografts, or metastatic tumours. After a careful review, a total of 928 PDAC patients from three microarrays and three RNA-sequencing datasets were selected for further analysis. The largest cohort (MTAB-6134, $\mathrm{n}=288$ ) was used as the training dataset. Five other cohorts, GSE21501 $(\mathrm{n}=102)$, GSE71729 $(\mathrm{n}=123)$, PACA-AU $(n=92)$, PACA-CA $(n=182)$, and TCGA $(n=141)$, were used for external validation. Detailed information about OS events and time were provided in all of the abovementioned datasets, whereas clinical DFS data were available in only four datasets, namely, MTAB-6134, PACA-AU, PACA-CA, and TCGA. In addition, a total of 48 frozen primary PDAC samples were collected at the Department of General Surgery of Ruijin Hospital from April 2012 to August 2018. The follow-up lasted until February 2019. Written informed consent was obtained from all patients. This study was conducted and approved in accordance with the Declaration of Helsinki, and the Ethics Committee of Ruijin Hospital affiliated with Shanghai Jiao Tong University approved the study. The baseline characteristics of PDAC patients enrolled in this study are listed in Additional file 1: Table S1.

\section{CSC-related genes identification}

The online software GEO2R (https://www.ncbi.nlm. nih.gov/geo/geo2r/) [25] was used to screen differentially expressed genes (DEGs) between four CSCs $(\mathrm{CD} 44+\mathrm{CD} 133+\mathrm{EPCAM}+)$ and four non-CSCs (CD44- CD133- EPCAM-) isolated from MIAPACA-2 PDAC cells in the GSE51971 dataset. DEGs with an adjusted $P$ value $<0.01$ and $|\log 2 \mathrm{FC}| \geq 2$ were identified as CSC-related genes.

\section{CSC-related gene signature establishment and validation} Univariate Cox regression analysis was applied to screen CSC-related genes significantly correlated with PDAC prognosis in three microarray datasets, namely GSE21501, GSE71729, and MTAB-6134. Genes with $\mathrm{P}<0.05$ in all three datasets were identified through Venn diagram (https://bioinfogp.cnb.csic.es/tools/venny/index .html.) The expression of selected genes in tumor and adjacent normal tissues were obtained from the GEPIA website (https://gepia.cancer-pku.cn/index.html).

The risk score of the gene signature was calculated as follows: risk score $=($ Coefficientgene $1 \times$ expression of gene 1$)+($ Coefficientgene $2 \times$ expression of 
gene 2$)+\cdots+($ Coefficientgenen $\times$ expression genen $)$. The relative coefficient of each gene was obtained from the univariate Cox regression analysis in the MTAB6134 cohort. Patients in both the training and validation cohorts were then classified into low- and high-risk groups based on the optimal cut-off value determined by X-Tile software [26]. Kaplan-Meier (K-M) survival curves were used to analyse the differences in survival time between low- and high-risk patients. Receiver operating characteristic (ROC) curves and calibration curves comparing the predicted and observed survival probabilities were employed to assess the predictive performance. The association of this signature with DFS was analysed in four cohorts, MTAB-6134, PACA-AU, PACA-CA, and TCGA. Moreover, ROC curves and the concordance index (C-index) were employed to compare the predictive accuracy of our signature with previously reported signatures, which included one 6-gene [18] and two 3 -gene signatures [21, 22].

\section{Nomogram based on the prognostic signature}

Univariate and multivariate Cox regression analyses were adopted to assess potential prognostic factors for OS. Parameters including HRs, 95\% confidence intervals (CIs), and $\mathrm{P}$ values were generated using the 'survival' package and visualized with the 'forestplot' package in R. Then, a nomogram composed of independent prognostic factors was constructed to predict the 1-, 2-, and 3-year survival probabilities using the 'rms' R package. Next, we compared the discriminative ability of this nomogram with that of traditional clinical indicators using a timedependent area under the curve (AUC) plotted by the 'timeROC' package in $\mathrm{R}$. The integrated discrimination improvement index (IDI) and net reclassification index (NRI) were calculated by the 'PredictABEL' R package to compare the predictive performance of the prognostic models before and after the inclusion of the risk signature. The 1-, 2-, and 3-year decision curve analysis (DCA) was used to evaluate the clinical validity of the nomogram [27].

\section{RNA extraction and quantitative real-time polymerase chain reaction ( $q R T-P C R$ )}

The total RNA of 48 PDAC samples (Ruijin cohort) was isolated with TRIzol reagent (Invitrogen, USA) and reverse-transcribed using the ReverTra Ace qPCR RT Kit (Toyobo, Japan). Real-time PCR was performed with an ABI 7900 instrument using SYBR Green (Toyobo). Quantitation was performed in triplicate. mRNA expression was calculated using the $2^{\Delta \Delta C T}$ method and normalized to glyceraldehyde-3-phosphate dehydrogenase (GAPDH). The primers for the amplified mRNAs are listed in Additional file 1: Table S2.

\section{Statistical analysis}

The statistical analysis and graphical work were done in the $\mathrm{R}$ environment (version 3.5.1). K-M survival curves were derived using the 'survival' package. ROC curves were plotted by the 'survivalROC' package. C-indices were calculated by the 'survcomp' package. Boxplots were depicted using the 'ggpubr' package. Samples with an OS or DFS of $<1$ month were excluded from the survival analyses. A two-sided log-rank $\mathrm{P}<0.05$ was considered significant.

\section{Results \\ CSC-related gene signature construction}

Figure 1 displays the overall study design and data analyses. The selection criteria p.adj $<0.01$ and $|\log 2 \mathrm{FC}|>2$ resulted in the identification of 334 CSC-related genes. According to the hazard ratios (HRs) from univariate Cox regression analysis, genes associated with better prognosis $(H R<1)$ were considered as protective genes and genes associated with worse prognosis $(\mathrm{HR}>1)$ were considered as risky genes. To improve accuracy, candidate genes were further filtered to exclude risky genes significantly downregulated in CSCs or protective genes significantly upregulated in CSCs. By utilizing Venn diagram, we identified four CSC-related genes (DCBLD2, GSDMD, PLOD2, PMAIP1) that were collectively correlated with unfavourable prognosis in three independent datasets and made up the prognostic signature. The HRs, 95\% CIs, and $\mathrm{p}$ values of the four genes are shown in Additional file 2: Fig. S1. In addition to being highly expressed in CSCs, the four identified genes were also highly expressed in cancer tissues (Additional file 2: Fig. S2), which suggested that these genes probably play important roles in PDAC progression. The risk score of each patient was calculated as follows: Risk score $=(0.31113 \times$ expression value of DCBLD2 $)+(0.293903 \times$ expression value of $\mathrm{GSDMD})+(0.514119 \times$ expression value of PLOD2 $)+(0.19192 \times$ expression value of PMAIP1).

\section{Performance assessment of the signature in predicting OS} We first applied the above formula to accessible datasets that contained both cancer and normal samples. PDAC tissues had a significantly higher risk score than adjacent normal tissues (Fig. 2a) and other subtypes of pancreatic cancer tissues (Fig. 2b). These findings indicate the hazardous role of the presence of this gene signature. We next investigated the relationship of risk score and patient prognosis in six independent PDAC cohorts. K-M survival curves estimated a significantly different OS between high-risk patients and low-risk patients (Fig. 2c). Then, we assessed the OS difference in high-, moderate-, and low-risk groups. The results 


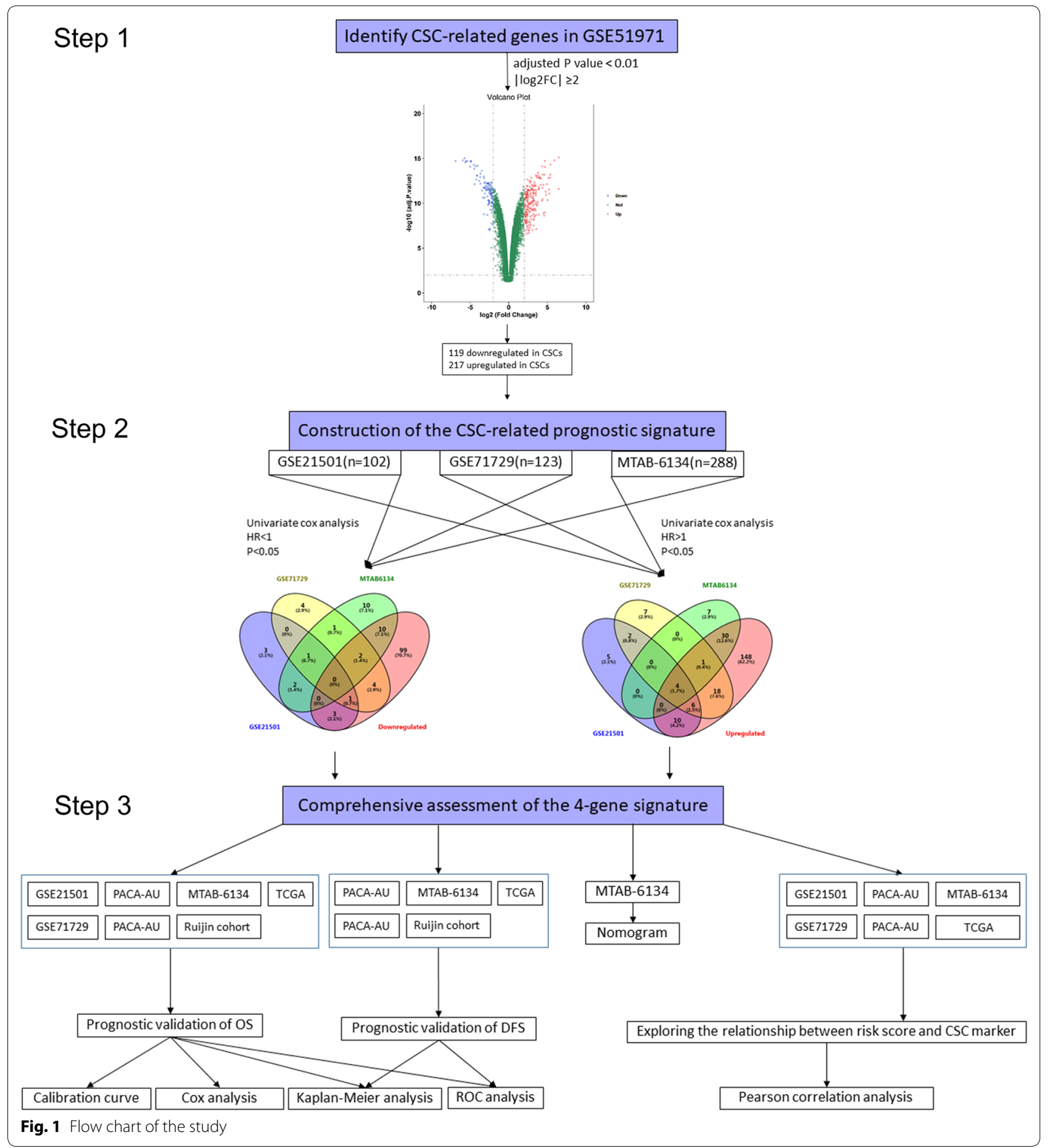

showed that the higher the risk category was, the lower the OS probability was (Fig. 2d).The calibration curves indicated that the 1-, 2- and 3-year survival probabilities predicted by this signature were in good agreement with the actual observations (Fig. 2e).
We further compared the robustness of the 4-gene model with previously reported models. The C-index of our model in the GSE21501, GSE71729, MTAB-6134, PACA-AU, PACA-CA and TCGA datasets was 0.59 (95\% CI, 0.52-0.67), 0.59 (95\% CI, 0.52-0.66), 0.65 (95\% 


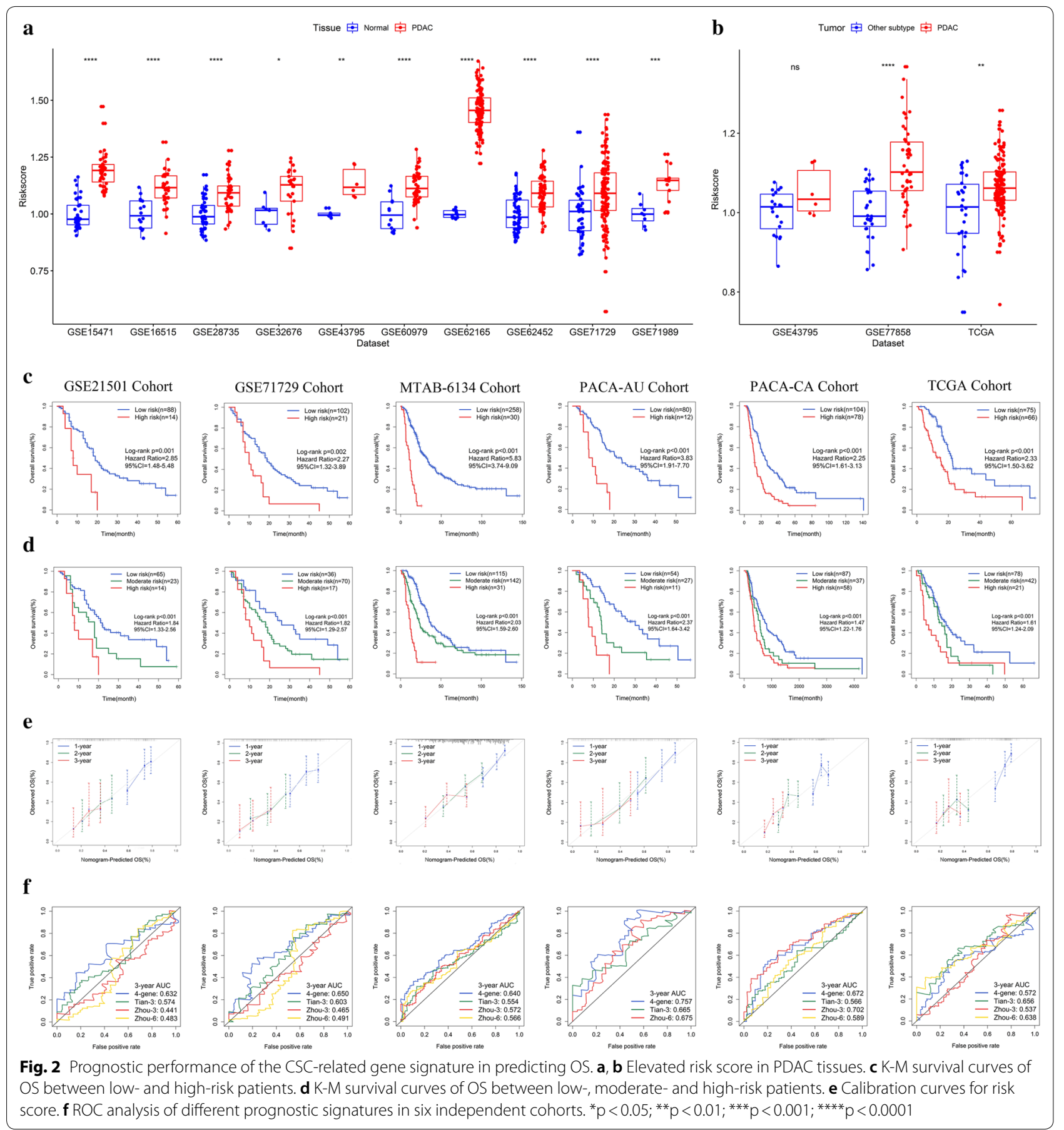

CI, 0.61-0.69), 0.69 (95\% CI, 0.62-0.75), 0.59 (95\% CI, 0.54-0.65), and 0.62 (95\% CI, 0.56-0.68), respectively, which is greater than that of previous models in these datasets except TCGA (Additional file 1: Table S3). This finding was not strange, given that TCGA is the dataset that generated these models. ROC analysis showed similar trends (Fig. 2f). The above results demonstrate that the CSC-related gene signature can better predict survival than other indicators.

Performance assessment of the signature in predicting DFS As with the results for OS, patients in the low-risk group had a significantly longer DFS than those in the high-risk group (MTAB-6134: $\mathrm{HR}=3.95,95 \%$ 

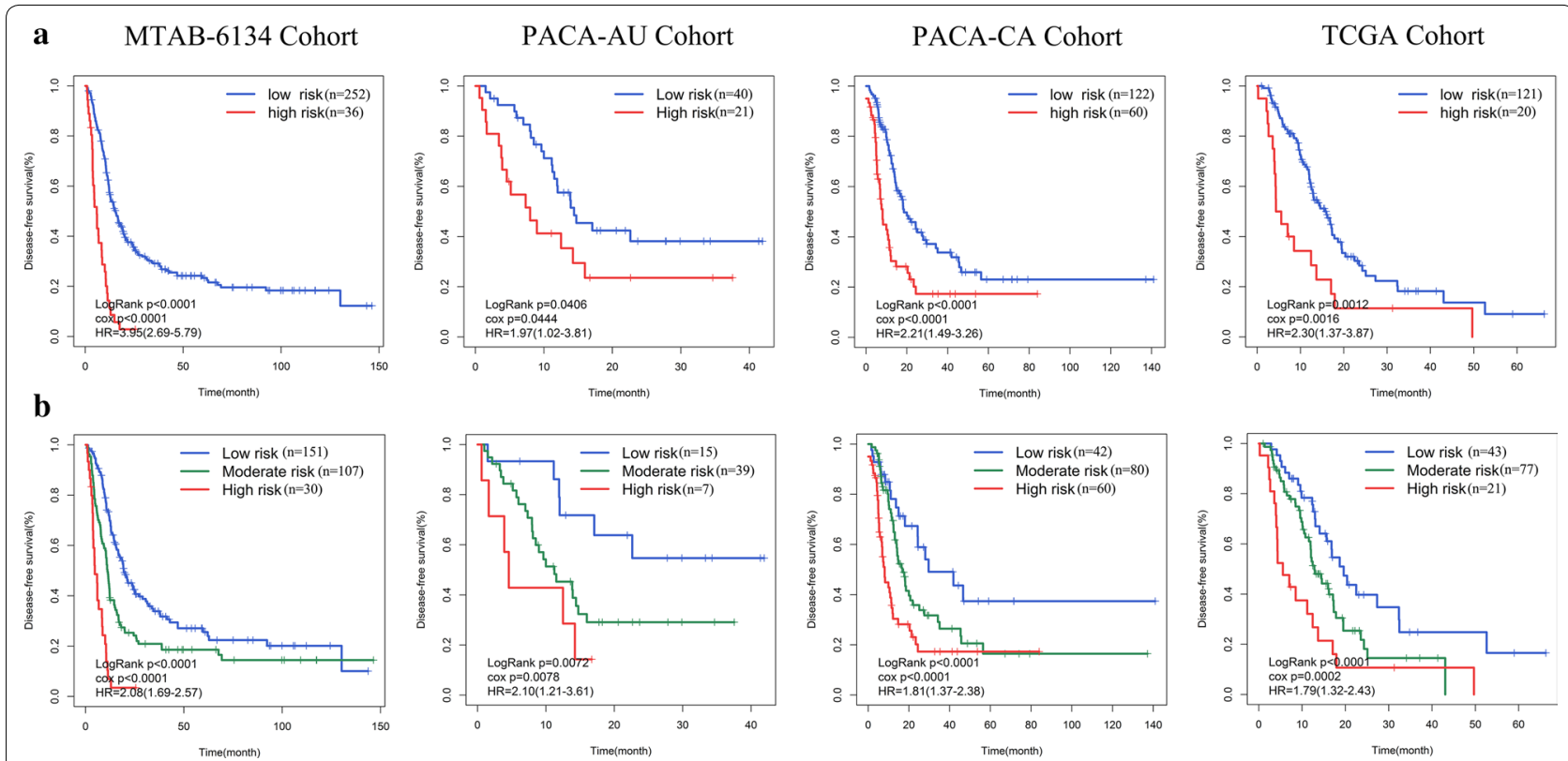

Fig. 3 Prognostic performance of the CSC-related gene signature in predicting DFS. a Kaplan-Meier curves estimating the DFS difference between low- and high-risk groups. b DFS difference among low-, moderate- and high-risk groups

$\mathrm{CI}=2.69-5.79, \mathrm{P}<0.0001 ; \mathrm{PACA}-\mathrm{AU}: \mathrm{HR}=1.97,95 \%$ $\mathrm{CI}=1.02-3.81, \mathrm{P}=0.0444 ; \mathrm{PACA}-\mathrm{CA}: \mathrm{HR}=2.21,95 \%$ $\mathrm{CI}=1.49-3.26, \quad \mathrm{P}<0.0001 ; \quad \mathrm{TCGA}: \quad \mathrm{HR}=2.30, \quad 95 \%$ $\mathrm{CI}=1.37-3.87, \mathrm{P}=0.0012$ ) (Fig. 3a). The differences in DFS among the high-, moderate-, and low-risk groups were next analysed. The results further confirmed that high risk score was significantly associated with short DFS (MTAB-6134: $\mathrm{HR}=2.08,95 \% \mathrm{CI}=1.69-2.57$, $\mathrm{P}<0.0001$; PACA-AU: $\mathrm{HR}=2.10,95 \% \mathrm{CI}=1.21-3.61$, $\mathrm{P}=0.0072$; PACA-CA: $\mathrm{HR}=1.81,95 \% \mathrm{CI}=1.37-2.38$, $\mathrm{P}<0.0001 ; \quad$ TCGA: $\mathrm{HR}=1.79,95 \% \mathrm{CI}=1.32-2.43$, $\mathrm{P}<0.0001$ ) (Fig. 3b). These results demonstrated that the CSC-related gene signature can be used as an effective prognostic indicator of DFS.

Validation of the prognostic performance in a local dataset To validate the above bioinformatic findings, we employed qPCR to detect the expression profiles of four genes in the Ruijin cohort. K-M curves showed that the signature effectively captured the survival differences in OS and DFS (Fig. 4a, b). Figure 4c, d shows that the signature showed high AUCs for OS prediction (1 year: 0.722 ; 2 years: 0.862 ) and DFS prediction (1 year: 0.798; 2 years: 0.785 ). Moreover, we observed significantly elevated expression of the four genes, except GSDMD (probably due to the limited sample size), in PDAC tissues (Fig. 4e).
Cox regression analyses of the signature prognostic value To verify the independent prognostic role of our model in OS prediction, univariate and multivariate Cox regression analyses were performed. Variables included gene signature and clinicopathological features. Univariate Cox analysis demonstrated that the prognostic value of the CSC-related gene signature was independent of other clinicopathological features in four independent cohorts (MTAB-6134: $\mathrm{HR}=1.89, \quad 95 \% \quad \mathrm{CI}=1.51-2.36, \quad \mathrm{P}<0.001 ; \quad \mathrm{PACA}-\mathrm{AU}:$ $\mathrm{HR}=1.93, \quad 95 \% \mathrm{CI}=1.30-2.86, \quad \mathrm{P}=0.001 ; \quad$ PACA-CA: $\mathrm{HR}=1.19,95 \% \mathrm{CI}=1.01-1.41, \mathrm{P}=0.036$; $\mathrm{TCGA}: \mathrm{HR}=1.38$, 95\% $\mathrm{CI}=1.06-1.81, \mathrm{P}=0.018$ ) (Fig. 5a). Multivariate analysis further confirmed the independent prognostic role of our model after adjusting for other clinical features in each cohort (Fig. 5b). Next, we evaluated association between the risk score and histological grade. Figure $5 c$ shows that the risk score was significantly higher in grade 3 and 4 patients than in grade 1 and 2 patients $(\mathrm{p}<0.05)$, indicating that a high risk score was associated with high malignancy.

\section{Nomogram based on the signature}

Nomogram is a quantitative method widely used for accurate assessment of patient survival. By adding up the corresponding scores of factors in the nomogram, clinician could obtain a predicted OS probability of individual patient. In this way, PDAC patients could be managed by more closely tailored treatments with the balance of 


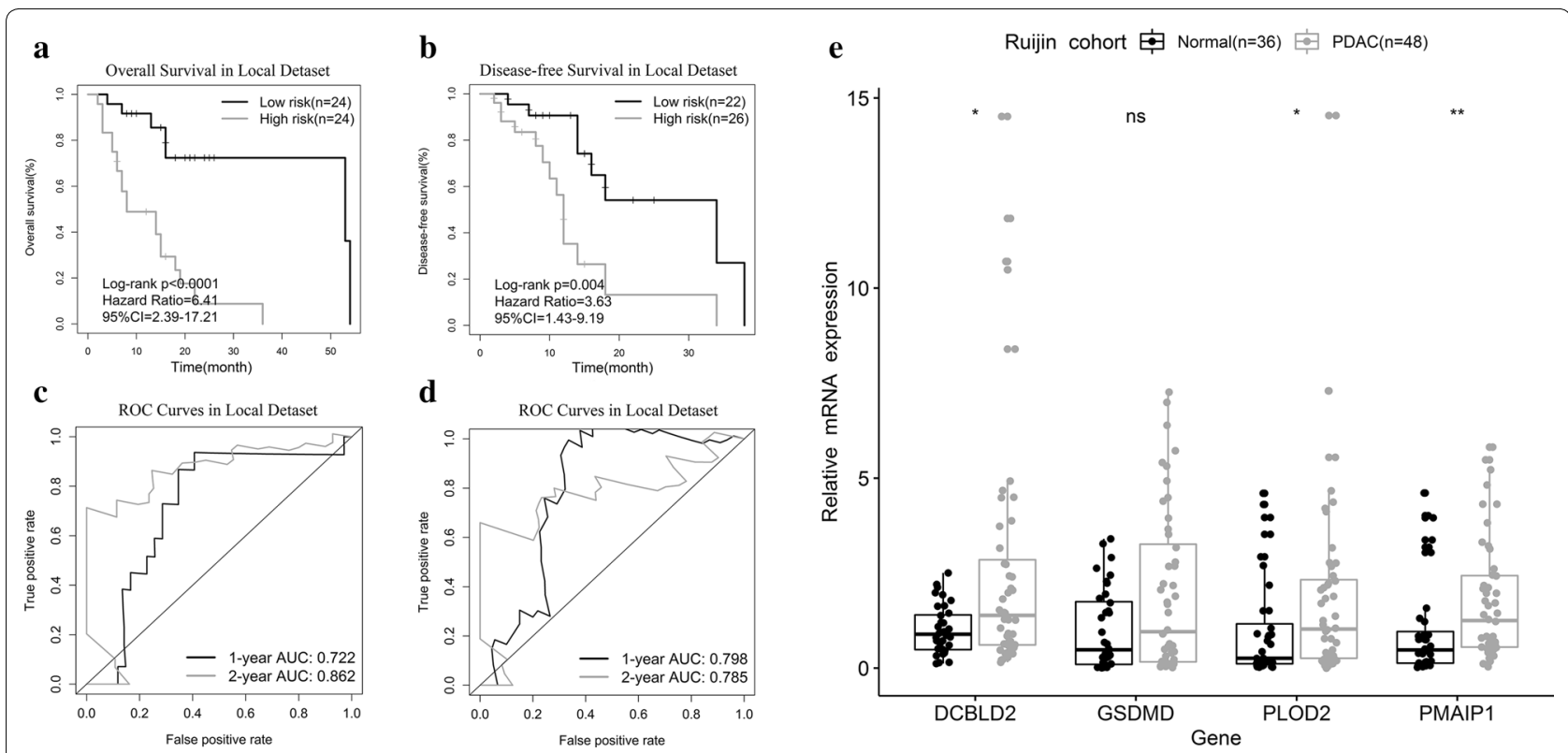

Fig. 4 Prognostic validation in a local dataset. a, b K-M curves of OS and DFS. c, d ROC curve analysis of the signature to predict 1- and 2-year OS and DFS. e The expression of four key genes in the Ruijin cohort. ${ }^{*} \mathrm{p}<0.05$; ${ }^{* *} \mathrm{p}<0.01$
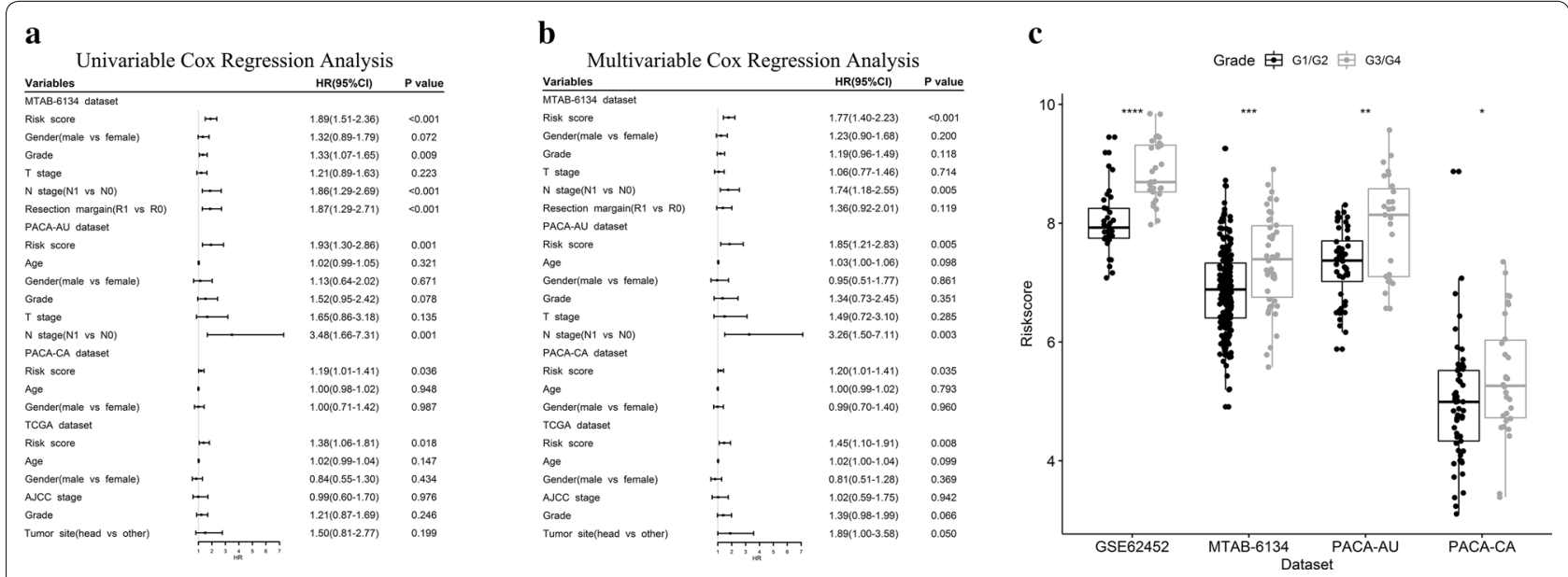

Fig. 5 Cox regression analyses of the signature in four datasets. a Univariate and $\mathbf{b}$ multivariate Cox regression analyses of risk score and clinical features. $\mathbf{c}$ Distribution of risk scores in PDAC patients stratified by histological grade. ${ }^{*} p<0.05 ;{ }^{* *} p<0.01 ;{ }^{* * *} p<0.001 ;{ }^{* * *} p<0.0001$

adverse effects and survival benefits. Risk score, grade, $\mathrm{N}$ stage, and resection margin were the variables included in the nomogram (Fig. 6a). K-M curves illustrated that the nomogram efficaciously distinguished patients with different OS and DFS (Fig. 6b, c). We next compared our signature to clinical prognostic indicators through AUC analysis. The results showed that the risk score had a higher dynamic AUC than grade, N stage, and resection margin over time in predicting OS and DFS (Fig. 6d, e). In other words, our model was more sensitive and specific than traditional clinical indicators for prognostic prediction. Furthermore, compared with the clinical model composed of grade, $\mathrm{N}$ stage, and resection margin, the predictive ability improved significantly when the risk score was included in the risk prediction model $(\mathrm{NRI}=0.236,95 \% \mathrm{CI}=-0.002-0.474, \mathrm{P}=0.052$; $\mathrm{IDI}=0.023,95 \% \mathrm{CI}=0.006-0.041, \mathrm{P}=0.009)$. The $\mathrm{DCA}$ of this nomogram for 1, 2, and 3 years is shown in Fig. $6 f$. The results showed that the constructed nomogram outperformed the strategies of treating all and treating none in predicting the survival probability within a limited range of threshold probabilities. 


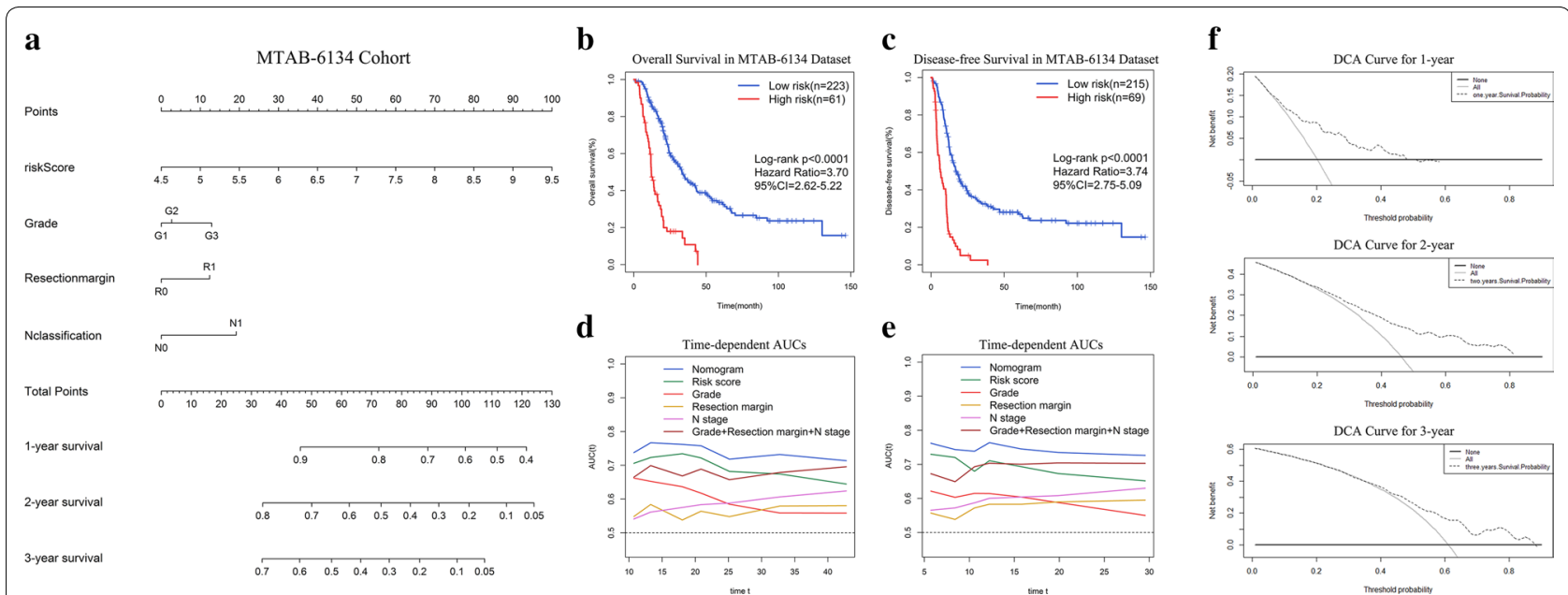

Fig. 6 A nomogram for prognostic prediction in PDAC. a Nomogram based on signature risk score, grade, N stage and resection margin for 1-, 2- and 3-year OS prediction. b, c K-M curves evaluating the OS and DFS between two groups defined by the nomogram. d, e Time-dependent AUC curves comparing the predictive abilities of prognostic factors for OS and DFS in the MTAB-6134 dataset. $\mathbf{f}$ 1-year, 2-year and 3-year DCA curves for signature

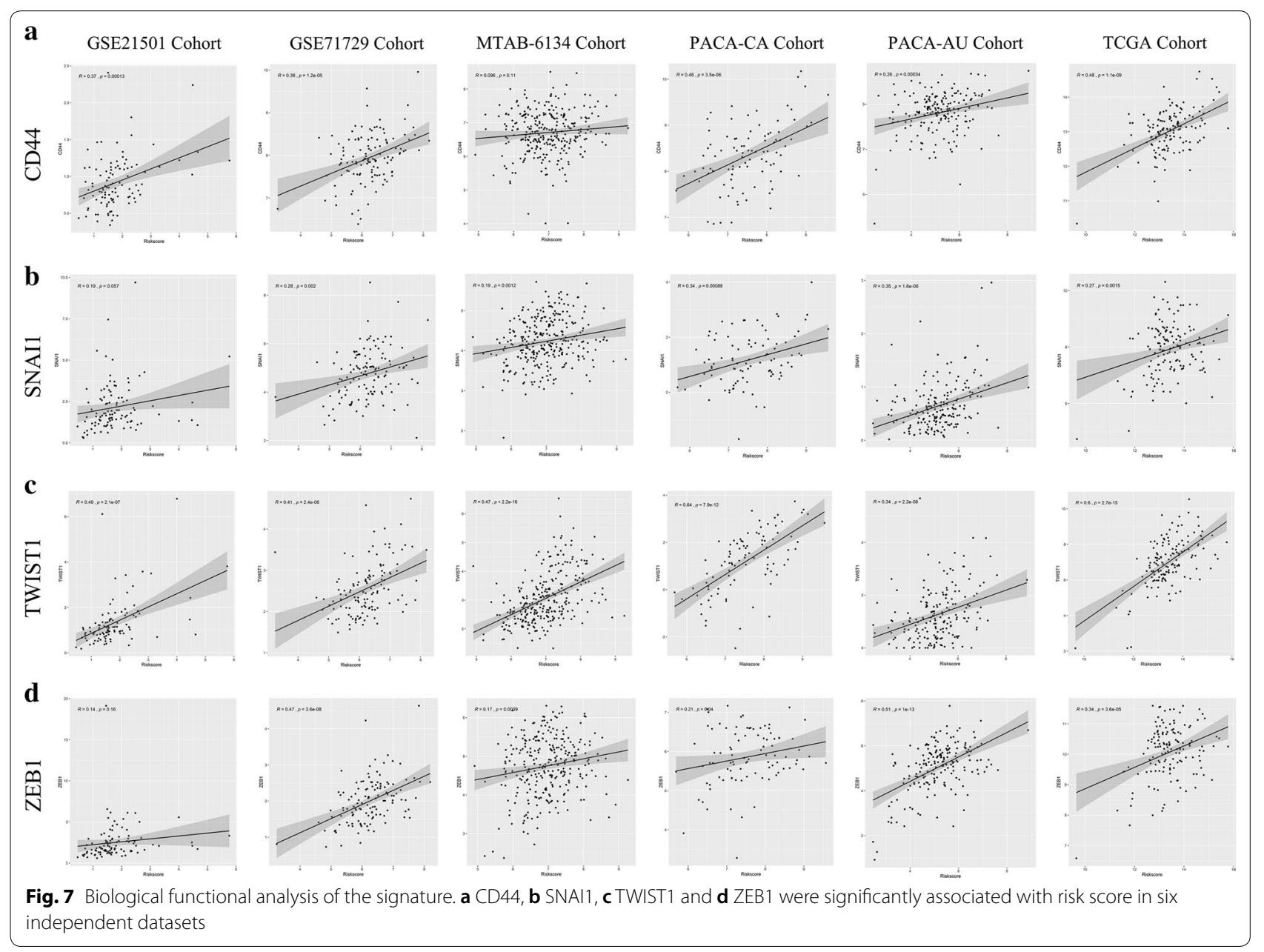




\section{Correlation of the signature with CSC-specific markers}

To clarify the potential functional characteristics of this model, we investigated the correlation of the risk score with a set of oncogenes that are involved in CSC formation and maintenance. Figure $7 \mathrm{a}$ shows that CD44, a CSC-specific marker, was significantly positively correlated with the risk score in the GSE21501 $(r=0.37$, $\mathrm{p}<0.001)$, GSE71729 $(\mathrm{r}=0.38, \mathrm{p}<0.001), \quad$ PACA-AU $(\mathrm{r}=0.48, \mathrm{p}<0.001)$, PACA-CA $(\mathrm{r}=0.26 . \mathrm{p}<0.001)$ and TCGA datasets $(r=0.48 . p<0.001)$. SNAI1, TWIST1, and ZEB1, three key regulators of the EMT process, showed the same trends in all six datasets (Fig. $7 \mathrm{~b}-\mathrm{d}$ ). Emerging evidence has confirmed that the activation of the EMT programme may give rise to CSCs. Similar results were also observed for SMO in the Hedgehog pathway, FZD7 in the WNT pathway, and NOTCH1 and $\mathrm{NOTCH} 2$ in the NOTCH pathway in multiple datasets (data not shown). These results collectively demonstrate that a high risk score might represent high CSC activity and enrichment, which could partly explain the negative association between the risk score and patient survival.

\section{Discussion}

PDAC has become a global public health problem because of its increasing incidence and high mortality [1]. The inherent chemo- and radio-resistant nature of this refractory disease has prompted attempts to define effective prognostic prediction models. In this study, we developed a robust 4-gene signature with cross-platform compatibility based on a panel of CSC-related genes. The proposed model exhibited better predictive performance for OS and DFS than several traditional clinical indicators, such as $\mathrm{N}$ stage and histological grade. Then, a nomogram integrating the risk signature and prognostic clinical factors was constructed for individualized survival assessment. Moreover, functional analysis revealed that the signature was positively associated with CSC activity.

Autophagy-, EMT-, and immune-related gene signatures of cancers have been extensively reported $[9,10$, 28]. However, few studies have analysed CSC expression profiles to construct a risk prediction model. CSCs are responsible for the therapy resistance, metastatic activity, and poor outcome of PDAC [29, 30]. We therefore employed CSC-related genes to build such a signature. In PDAC, most previously developed molecular signatures have been derived from a single TCGA-PAAD training set. These models have minimal overlap, partly due to the heterogeneity of TCGA data sources and different statistical methods. In this study, only the common genes between three independent microarray datasets were eligible for inclusion in the final signature. This ensured the construction of a more precise signature than that generated from a single dataset. Moreover, the TCGAPAAD cohort comprised approximately 25-35 nonPDAC samples, such as neuroendocrine neoplasms and intraductal papillary neoplasms. However, these uncommon pancreatic malignancies displayed completely different molecular profiles and clinical outcomes from those of classical PDAC. This suggested that inclusion of such tumours in the training set can lead to inaccurate conclusions about prognostic performance [31]. To avoid repeating this mistake, non-PDAC samples were strictly filtered out in the current study.

During the construction of the gene signature, we initially identified four genes (DCBLD2, GSDMD, PLOD2, and PMAIP1) related to the OS of PDAC patients using a Venn diagram based on the result of univariate Cox regression analysis. From this, a 4-gene signature was developed. Survival analyses indicated that the signature distinguished PDAC patients with significantly different OS and DFS rates. ROC analyses showed that the signature had higher AUC values than previous models and clinical indicators, indicating the better survival prediction of our model.

Among those four genes, PLOD2 and PMAIP1 were previously reported to be closely related to PDAC. PLOD2 is essential for the formation of normal mature collagen [32]. High PLOD2 expression is associated with poor outcomes in patients with liver [33], breast [34], and lung cancer [35]. In PDAC, increased expression of PLOD2 under hypoxic conditions promotes cell motility and thus facilitates tumour progression [36]. PMAIP1 is a crucial gene for the activation of caspases and apoptosis [37]; it has been identified as a candidate tumour suppressor gene that is frequently downregulated in pancreatic cancer [38]. However, its prognostic value has not been explored to date. DCBLD2 and GSDMD have considerable tumour-specific effects, but their roles in PDAC development remain unclear. DCBLD2 is overexpressed in glioblastoma, colorectal cancer, and lung cancer [3941], and it is strongly associated with tumour migration and invasion. GSDMD plays an important role in the regulation of pyroptosis and sensitivity to cancer therapy [42]. GSDMD downregulation contributes to the occurrence and proliferation of gastric cancer [43], whereas in non-small-cell lung cancer, its upregulation is correlated with poor prognosis [44].

High biological heterogeneity poses a challenge to prognostic assessment and treatment decisions in PDAC. In the context of precision medicine, one of the top priorities is to develop a precise prognostic model that can lead to a more tailored therapeutic strategy for individual patients by considering their molecular heterogeneity. The current staging system only considers anatomical factors and cannot capture personalized 
genetic characteristics [45]. Thus, we established a risk signature based on the individual expression values of four key genes. The use of fewer genes than previous signatures makes the present model more applicable in the clinic. We hope that this model can be translated into a fast detection kit based on PCR. In this way, the signature can provide potential value for making personalized treatments and saving public health resources. Moreover, validation in a large cohort including Americans, Europeans, and our local Asian population reinforces the signature's credibility and reveals the potential of the model for application in patients of different races and nationalities.

The present study had several limitations. First, detailed clinical information on chemo-radiotherapeutic treatments was not available. Thus, our risk signature cannot provide information on the individual therapeutic benefits of conventional treatments in each risk group. Second, the present study was based on retrospective data and has not been validated in prospective studies. Third, although the four genes were highly expressed in both cancer stem cells and cancer tissues, they are not confirmed targets in PDAC treatment. Additional in vivo and in vitro experiments are necessary to identify the biological roles of these genes. Fourth, the validation datasets were relatively small; therefore, the present findings need to be validated in a larger cohort.

In conclusion, we used CSC expression profiles to construct a practical four-gene signature and demonstrated that this signature could serve as a powerful predictor of OS and DFS in PDAC. The signature may provide reliable guidance and improved precision for available treatment applications. However, the predictive ability and clinical validity of the signature need to be further tested in larger cohorts, and the mechanisms connecting the four genes to poor prognosis need to be clarified.

\section{Conclusions}

We established a novel four-gene signature based on CSC-related genes that could serve as a powerful prognostic tool in PDAC.

\section{Supplementary information}

Supplementary information accompanies this paper at https://doi. org/10.1186/s12967-020-02527-1.

Additional file 1: Table S1. Clinicopathological characteristics of patients involved in the study. Table S2. Quantitative real-time PCR primer sequences. Table S3. The C-index of prognostic signatures in six independent datasets.

Additional file 2: Figure S1. Univariate Cox regression analyses of the four genes in three independent dataset. Figure $\mathbf{5 2}$. The expression of the four genes in cancer tissues and normal tisssues.
Abbreviations

PDAC: Pancreatic ductal adenocarcinoma; CSC: Cancer stem cell; EMT: Epithelial-mesenchymal transition; OS: Overall survival; DFS: Disease-free survival; ROC: Receiver operating characteristic; AUC: Area under the curve; qRT-PCR: Quantitative real-time reverse transcription polymerase chain reaction; HR: Hazard ratio; 95\% Cl: 95\% Confidence interval; GEO: Gene Expression Omnibus; TCGA: The Cancer Genome Atlas; TNM: Tumour, node, metastasis; ICGC: International Cancer Genome Consortium; RMA: Robust multi-array averaging; DEGs: Differentially expressed genes.

\section{Acknowledgements}

We thank the Department of General Surgery of Ruijin Hospital for providing the frozen tissue specimens. We thank Nature Research Editing Service (https ://bit.ly/NRES-HS) for editing this manuscript. We thank Dr. Yang Ma for help in statistical and bioinformatical review. This work was supported by grants from National Natural Science Foundation of China (81672325 and 81802316).

\section{Authors' contributions}

All authors contributed to the study conception and design. Material preparation, data collection and analysis were performed by ZF, MS and KL. The first draft of the manuscript was written by ZF and all authors commented on previous versions of the manuscript. Statistical method was reviewed by YM. All authors read and approved the final manuscript.

\section{Funding}

We thank the Department of General Surgery of Ruijin Hospital for providing the frozen tissue specimens. We thank Nature Research Editing Service (https ://bit.ly/NRES-HS) for editing this manuscript. We thank Dr. Yang Ma for help in statistical and bioinformatical review. This work was supported by grants from National Natural Science Foundation of China (81672325 and 81802316).

\section{Availability of data and materials}

The datasets generated during and/or analysed during the current study are available in the Gene Expression Omnibus (https://www.ncbi.nlm.nih.gov/ geo/), ArrayExpress (https://www.ebi.ac.uk/arrayexpress/), International Cancer Genome Consortium (https://icgc.org/), and The Cancer Genome atlas (https ://cancergenome.nih.gov/) databases. $\mathrm{R}$ code is available upon request.

\section{Ethics approval and consent to participate}

The Ethics Committee of Ruijin Hospital affiliated with Shanghai Jiao Tong University approved the study. Informed consent was obtained from all individual participants included in the study.

\section{Consent for publication}

Consent to publish has been obtained from all authors.

\section{Competing interests}

The authors declare that they have no competing interests.

\section{Author details}

${ }^{1}$ Department of General Surgery, Pancreatic Disease Center, Research Institute of Pancreatic Diseases, Ruijin Hospital, Shanghai Jiaotong University School of Medicine, Shanghai, China. ${ }^{2}$ State Key Laboratory of Oncogenes and Related Genes, Institute of Translational Medicine, Shanghai Jiaotong University, Shanghai, China.

Received: 16 March 2020 Accepted: 15 September 2020

Published online: 21 September 2020

References

1. Siegel RL, Miller KD, Jemal A. Cancer statistics, 2019. CA Cancer J Clin. 2019;69(1):7-34.

2. Han H, Von Hoff DD. SnapShot: pancreatic cancer. Cancer Cell. 2013;23(3):424-424.e1.

3. Kamisawa T, Wood LD, Itoi T, Takaori K. Pancreatic cancer. Lancet. 2016;388(10039):73-85

4. Oettle H, Post S, Neuhaus P, Gellert K, Langrehr J, Ridwelski K, et al. Adjuvant chemotherapy with gemcitabine vs observation in patients undergoing 
curative-intent resection of pancreatic cancer: a randomized controlled trial. JAMA. 2007;297(3):267-77.

5. El Hassouni B, Li Petri G, Liu DSK, Cascioferro S, Parrino B, Hassan W, et al. Pharmacogenetics of treatments for pancreatic cancer. Expert Opin Drug Metab Toxicol. 2019;15(6):437-47.

6. Allen PJ, Kuk D, Castillo CF-D, Basturk O, Wolfgang CL, Cameron JL, et al. Multi-institutional Validation Study of the American Joint Commission on Cancer (8th Edition) changes for T and $\mathrm{N}$ staging in patients with pancreatic adenocarcinoma. Ann Surg. 2017:265(1):185-91.

7. Diwakarla C, Hannan K, Hein N, Yip D. Advanced pancreatic ductal adenocarcinoma-complexities of treatment and emerging therapeutic options. World J Gastroenterol. 2017;23(13):2276-85.

8. Zhou Z, Mo S, Dai W, Ying Z, Zhang L, Xiang W, et al. Development and validation of an autophagy score signature for the prediction of post-operative survival in colorectal cancer. Front Oncol. 2019;9:878

9. Cao R, Yuan L, Ma B, Wang G, Qiu W, Tian Y. An EMT-related gene signature for the prognosis of human bladder cancer. J Cell Mol Med. 2019:24(1):605-17.

10. Zeng F, Liu X, Wang K, Zhao Z, Li G. Transcriptomic profiling identifies a DNA repair-related signature as a novel prognostic marker in lower grade gliomas. Cancer Epidemiol Biomarkers Prev. 2019;28(12):2079-86.

11. Simeone DM. Pancreatic cancer stem cells: implications for the treatment of pancreatic cancer. Clin Cancer Res. 2008;14(18):5646-8.

12. Hermann PC, Huber SL, Herrler T, Aicher A, Ellwart JW, Guba M, et al. Distinct populations of cancer stem cells determine tumor growth and metastatic activity in human pancreatic cancer. Cell Stem Cell. 2007;1(3):313-23.

13. Wang VMY, Ferreira RMM, Almagro J, Evan T, Legrave N, Zaw Thin M, et al. CD9 identifies pancreatic cancer stem cells and modulates glutamine metabolism to fuel tumour growth. Nat Cell Biol. 2019;21(11):1425-35.

14. Lee SH, Kim H, Hwang J-H, Shin E, Lee HS, Hwang DW, et al. CD24 and S100A4 expression in resectable pancreatic cancers with earlier disease recurrence and poor survival. Pancreas. 2014;43(3):380-8.

15. Liu Y, WU T, Lu D, Zhen J, Zhang L. CD44 overexpression related to lymph node metastasis and poor prognosis of pancreatic cancer. Int J Biol Markers. 2018:33(3):308-13.

16. Li X, Zhao H, Gu J, Zheng L. Prognostic value of cancer stem cell marker CD133 expression in pancreatic ductal adenocarcinoma (PDAC): a systematic review and meta-analysis. Int J Clin Exp Pathol. 2015;8(10):12084-92.

17. Zhou C, Zhao Y, Yin Y, Hu Z, Atyah M, Chen W, et al. A robust 6-mRNA signature for prognosis prediction of pancreatic ductal adenocarcinoma. Int J Biol Sci. 2019;15(11):2282-95.

18. Liao X, Huang K, Huang R, Liu X, Han C, Yu L, et al. Genome-scale analysis to identify prognostic markers in patients with early-stage pancreatic ductal adenocarcinoma after pancreaticoduodenectomy. Onco Targets Ther. 2017;10:4493-506

19. Haider S, Wang J, Nagano A, Desai A, Arumugam P, Dumartin L, et al. A multi-gene signature predicts outcome in patients with pancreatic ductal adenocarcinoma. Genome Med. 2014;6(12):105.

20. Stratford JK, Bentrem DJ, Anderson JM, Fan C, Volmar KA, Marron JS, et al. A six-gene signature predicts survival of patients with localized pancreatic ductal adenocarcinoma. PLoS Med. 2010;7(7):e1000307.

21. Zhou X, Liao X, Wang X, Huang K, Yang C, Yu T, et al. Clinical significance and prospective molecular mechanism of $\mathrm{C}-\mathrm{C}$ motif chemokine receptors in patients with early-stage pancreatic ductal adenocarcinoma after pancreaticoduodenectomy. Oncol Rep. 2019:42(5):1856-68.

22. Tian G, Li G, Liu P, Wang Z, Li N. Glycolysis-based genes associated with the clinical outcome of pancreatic ductal adenocarcinoma identified by the cancer genome atlas data analysis. DNA Cell Biol. 2020;39(3):417-27.

23. Peran I, Madhavan S, Byers SW, McCoy MD. Curation of the pancreatic ductal adenocarcinoma subset of the cancer genome atlas is essential for accurate conclusions about survival-related molecular mechanisms. Clin Cancer Res. 2018:24(16):3813-9.

24. Irizarry RA, Hobbs B, Collin F, Beazer-Barclay YD, Antonellis KJ, Scherf U, et al. Exploration, normalization, and summaries of high density oligonucleotide array probe level data. Biostatistics. 2003;4(2):249-64.

25. Barrett T, Wilhite SE, Ledoux P, Evangelista C, Kim IF, Tomashevsky M, et al. NCBI GEO: archive for functional genomics data sets-update. Nucleic Acids Res. 2013:41:D991-D99595.

26. Camp RL, Dolled-Filhart M, Rimm DL. X-tile: a new bio-informatics tool for biomarker assessment and outcome-based cut-point optimization. Clin Cancer Res. 2004:10(21):7252-9.
27. Vickers AJ, Elkin EB. Decision curve analysis: a novel method for evaluating prediction models. Med Decis Making. 2006;26(6):565-74.

28. Yang $S, W u Y$, Deng Y, Zhou L, Yang P, Zheng Y, et al. Identification of a prognostic immune signature for cervical cancer to predict survival and response to immune checkpoint inhibitors. Oncoimmunology. 2019;8(12):e1659094.

29. Lonardo E, Hermann PC, Mueller M-T, Huber S, Balic A, Miranda-Lorenzo I, et al. Nodal/Activin signaling drives self-renewal and tumorigenicity of pancreatic cancer stem cells and provides a target for combined drug therapy. Cell Stem Cell. 2011;9(5):433-46.

30. Hermann PC, Trabulo SM, Sainz B, Balic A, Garcia E, Hahn SA, et al. Multimodal treatment eliminates cancer stem cells and leads to long-term survival in primary human pancreatic cancer tissue xenografts. PLOS ONE. 2013;8(6):e66371.

31. Nicolle R, Raffenne J, Paradis V, Couvelard A, de Reynies A, Blum Y, et al. Prognostic biomarkers in pancreatic cancer: avoiding errata when using the TCGA dataset. Cancers. 2019;11(1):126.

32. van der Slot AJ, Zuurmond A-M, Bardoel AFJ, Wijmenga C, Pruijs HEH, Sillence DO, et al. Identification of PLOD2 as telopeptide lysyl hydroxylase, an important enzyme in fibrosis. J Biol Chem. 2003;278(42):40967-72.

33. Noda T, Yamamoto H, Takemasa I, Yamada D, Uemura M, Wada H, et al. PLOD2 induced under hypoxia is a novel prognostic factor for hepatocellular carcinoma after curative resection. Liver Int. 2012;32(1):110-8.

34. Gilkes DM, Bajpai S, Wong CC, Chaturvedi P, Hubbi ME, Wirtz D, et al. Procollagen lysyl hydroxylase 2 is essential for hypoxia-induced breast cancer metastasis. Mol Cancer Res. 2013;1 1(5):456-66.

35. Chen Y, Terajima M, Yang Y, Sun L, Ahn Y-H, Pankova D, et al. Lysyl hydroxylase 2 induces a collagen cross-link switch in tumor stroma. J Clin Invest. 2015;125(3):1147-62.

36. Sada M, Ohuchida K, Horioka K, Okumura T, Moriyama T, Miyasaka Y, et al. Hypoxic stellate cells of pancreatic cancer stroma regulate extracellular matrix fiber organization and cancer cell motility. Cancer Lett. 2016;372(2):210-8.

37. Villunger A, Michalak EM, Coultas L, Müllauer F, Böck G, Ausserlechner MJ, et al. p53- and drug-induced apoptotic responses mediated by $\mathrm{BH} 3$-only proteins puma and noxa. Science. 2003;302(5647):1036-8.

38. Ishida M, Sunamura M, Furukawa T, Lefter LP, Morita R, Akada M, et al. The PMAIP1 gene on chromosome 18 is a candidate tumor suppressor gene in human pancreatic cancer. Dig Dis Sci. 2008;53(9):2576-82.

39. Feng H, Lopez GY, Kim CK, Alvarez A, Duncan CG, Nishikawa R, et al. EGFR phosphorylation of DCBLD2 recruits TRAF6 and stimulates AKT-promoted tumorigenesis. J Clin Invest. 2014:124(9):3741-56.

40. Pagnotta SM, Laudanna C, Pancione M, Sabatino L, Votino C, Remo A, et al. Ensemble of gene signatures identifies novel biomarkers in colorectal cancer activated through PPARY and TNFa signaling. PLOS ONE. 2013;8(8):e72638

41. Koshikawa K, Osada H, Kozaki K-I, Konishi H, Masuda A, Tatematsu Y, et al. Significant up-regulation of a novel gene, CLCP1, in a highly metastatic lung cancer subline as well as in lung cancers in vivo. Oncogene. 2002;21(18):2822-8.

42. Wang Y-Y, Liu X-L, Zhao R. Induction of pyroptosis and its implications in cancer management. Front Oncol. 2019;9:971.

43. Wang WJ, Chen D, Jiang MZ, Xu B, Li XW, Chu Y, et al. Downregulation of gasdermin $D$ promotes gastric cancer proliferation by regulating cell cyclerelated proteins. J Dig Dis. 2018;19(2):74-83.

44. Gao J, Qiu X, Xi G, Liu H, Zhang F, LvT, et al. Downregulation of GSDMD attenuates tumor proliferation via the intrinsic mitochondrial apoptotic pathway and inhibition of EGFR/Akt signaling and predicts a good prognosis in non-small cell lung cancer. Oncol Rep. 2018;40(4):1971-84.

45. van Roessel S, Kasumova GG, Verheij J, Najarian RM, Maggino L, de Pastena $M$, et al. International Validation of the Eighth Edition of the American Joint Committee on Cancer (AJCC) TNM Staging System in Patients With Resected Pancreatic Cancer. JAMA Surg. 2018;153(12):e183617.

\section{Publisher's Note}

Springer Nature remains neutral with regard to jurisdictional claims in published maps and institutional affiliations. 